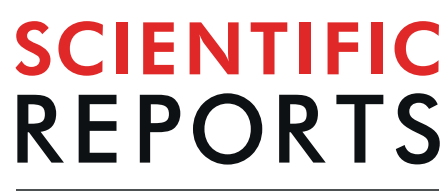

natureresearch

\title{
Broad and Efficient Control of Klebsiella Pathogens by Peptidoglycan-Degrading and Pore- Forming Bacteriocins Klebicins
}

\author{
Erna Denkovskienè $\dot{1}^{1}$ Šarūnas Paškevičius ${ }^{1,2}$, Audrius Misiūnas ${ }^{1}$, Benita Stočkūnaitè ${ }^{1}$, \\ Urtė Starkevič ${ }^{1}$, Astra Vitkauskienè ${ }^{3}$, Simone Hahn-Löbmann ${ }^{4}$, Steve Schulz ${ }^{4}$, \\ Anatoli Giritch ${ }^{4}$, Yuri Gleba ${ }^{4}$ \& Aušra Ražanskienè ${ }^{1 *}$
}

Gram-negative bacteria belonging to the genus Klebsiella are important nosocomial pathogens, readily acquiring resistance to all known antibiotics. Bacteriocins, non-antibiotic antibacterial proteins, have been earlier proposed as potential therapeutic agents for control of other Gram-negative species such as Escherichia, Pseudomonas and Salmonella. This study is the first report describing pore-forming and peptidoglycan-degrading bacteriocins klebicins from Klebsiella. We have identified, cloned, expressed in plants and characterized nine pore-forming and peptidoglycan-degrading bacteriocins from different Klebsiella species. We demonstrate that klebicins can be used for broad and efficient control of 101 of the 107 clinical isolates representing five Klebsiella species, including multi-drug resistant pathovars and pathovars resistant to carbapenem antibiotics.

Klebsiellae are nonmotile, rod-shaped, Gram-negative bacteria, encased by a polysaccharide capsule providing resistance against many host defense mechanisms ${ }^{1}$. Three species in the genus Klebsiella are commonly associated with illness in humans: K. pneumoniae, K. oxytoca, and K. granulomatis. Recently it was discovered that two more Klebsiella species, K. variicola and K. quasipneumoniae, also can cause deadly infections ${ }^{2}$.

Klebsiella is an important pathogen in nosocomial infections and is responsible for many clinical syndromes including pneumonia, bacteremia, thrombophlebitis, urinary tract infection, cholecystitis, diarrhea, upper respiratory tract infection, wound infection, osteomyelitis, and meningitis ${ }^{3}$. The use of invasive devices, use of respiratory support equipment, of urinary catheters, and treatment by antibiotics are risk factors causing potential infection ${ }^{4}$. The principal pathogenic reservoirs for infection are the gastrointestinal tract of patients and the hands of hospital personnel ${ }^{1}$.

Klebsiella pneumoniae is one of the six ESKAPE pathogens (Enterococcus faecium, Staphylococcus aureus, Klebsiella pneumoniae, Acinetobacter baumannii, Pseudomonas aeruginosa, Enterobacter spp.) causing hospital infections, which readily developed resistance to antibiotics ${ }^{5}$. In 2016 an outbreak of nosocomial pneumonia was reported in China, where five surgical patients died from infection with carbapenem-resistant K. pneumoniae (CRKP) $\mathrm{ST}_{11}$ strains that had acquired a virulence plasmid. Such new strains are simultaneously hypervirulent, multidrug resistant, and transmissible and could pose a serious threat to public health ${ }^{6}$.

In light of the ever-increasing drug resistance shown by many pathogens, the development of a new generation of antimicrobial substances is urgently needed, in particular to control Gram-negative bacteria. An alternative approach to antimicrobial treatment is to use the well-characterized species-specific colicin-like bacteriocins which are produced by a wide range of Gram-negative bacteria ${ }^{7}$. The potential use of such bacteriocins as narrow spectrum antimicrobials, in particular E. coli colicins and $P$. aeruginosa pyocins, have been explored in several studies $^{8-13}$.

Colicin-like bacteriocins, first described in E. coli, kill only closely related bacteria, belonging to the same species or genera. Their mechanisms of action are diverse, including pore-formation, DNAse or RNAse activity,

\footnotetext{
${ }^{1}$ Nomads UAB, Geležinio vilko 29A, LT-01112, Vilnius, Lithuania. ${ }^{2}$ Vilnius University, Institute of Biotechnology, Sauletekio al. 7, LT-10257, Vilnius, Lithuania. ${ }^{3}$ Lithuanian University of Health Sciences, Department of Laboratory Medicine, Eivenių g. 2, LT-50161, Kaunas, Lithuania. ${ }^{4}$ Nomad Bioscience GmbH, Biozentrum Halle, Weinbergweg 22, D-06120, Halle (Saale), Germany. *email: ausra@nomadsbio.It
} 


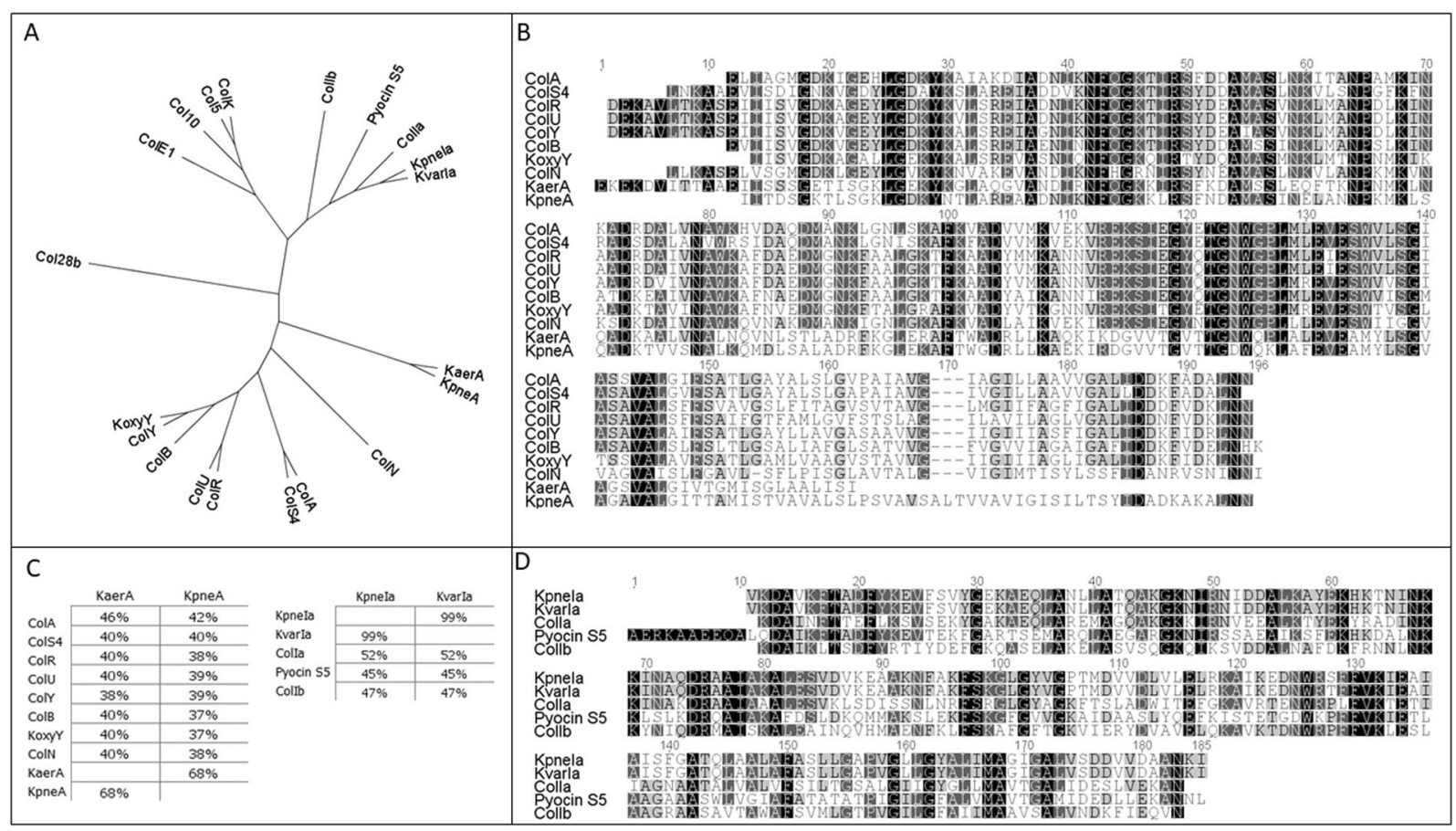

Figure 1. Sequence analysis of pore forming domain-containing colicins and their Klebsiella homologues. (A) Phylogenetic tree of predicted pore-forming domains (pfam01024; Geneious tree builder) of pore-forming colicins, pyocin S5 and klebicins. KpneA (SAV78255.1), KaerA (WP_063414841.1), KoxyY (WP_024273778), KvarIa (KDL88409), KpneIa (BAS34675), Col28B (CAA44310.1), ColE1 (AAA87379.1), Col10 (CAA57998.1), Col5 (CAA61102.1), ColK (Q47502.1), ColIb (AAA23188.1), Pyocin S5 (WP_003115311), ColIa (WP_001283344.1), Col N (P08083.1), ColA (P04480.1), ColS4 (CAB46008.1), ColR (AGV40809.1), ColU (CAĀ72509.1), ColB (P05819.3), ColY (AAF82683.1). (B) Clustal W amino acid sequence alignment of pore-forming domains of colicin A-like proteins. (C) Amino acid identity between pore-forming colicins and klebicins (Pfam14859). (D) Clustal W amino acid sequence alignment of pore-forming domains of colicin Ia-like proteins.

or disruption of peptidoglycan biosynthesis in the periplasm. The narrow target range of colicins has been shown to be due to the presence of specific receptors at the surface of the sensitive strains to which colicin binds before killing. The three steps of colicin action have been described: the colicin molecule causes killing after binding to a specific receptor on the outer membrane and being translocated through the cell envelope by either the Tol or TonB machinery to its target. All colicins are organized into three domains in relation with the three steps of their action: reception, translocation, and killing. Binding to specific receptors located in the outer membrane, translocation across the cell envelope, and cytotoxic activities are dependent on the central, N-terminal, and C-terminal domains, respectively ${ }^{14}$.

So far, the Klebsiella colicin-like bacteriocins, klebicins, have received very little attention and only few studies have been published, all describing klebicins with presumed nuclease activity ${ }^{15-17}$. Several putative ColM-type bacteriocins have been recently identified in silico in Klebsiella genomes ${ }^{18}$. We describe here several new pore-forming and peptidoglycan-degrading bacteriocins from different Klebsiella species and demonstrate that they can be used for broad and efficient control of Klebsiella pathogens, in particular multi-drug resistant pathovars.

\section{Results}

Identification and analysis of klebicin sequences. Putative Klebsiella bacteriocin amino acid sequences were retrieved from NCBI by BLAST search using as queries ColM (pfam 14859) and pore forming domains of ColA, ColIa and ColU (pfam01024). After the analysis of BLAST results we have selected the five most divergent pore-forming domain containing proteins from various Klebsiella species (K. pneumoniae SAV78255.1, K. aerogenes WP_063414841.1, K. oxytoca WP_024273778, K. variicola KDL88409, K. pneumoniae BAS34675) and four colicin M-like putative bacteriocins (K. pneumoniae EWD35590.1, Klebsiella sp. WP_047066220, K. variicola CTQ17225.1, K. aerogenes WP_015367360.1). The predicted pore-forming domain sequences of these putative bacteriocins were subjected to Clustal W amino acid sequence alignment and Geneious tree builder analysis along with all pore-forming domains of known pore-forming colicins and pyocin S5. Two major groups of Klebsiella pore-forming domain-containing proteins can be distinguished: K. pneumoniae BAS34675 and K. variicola KDL88409 are most related to colicin Ia, and K. aerogenes WP_063414841, K. pneumoniae SAV78255 and K. oxytoca WP_024273778 belong to the group of proteins similar to colicin A (Fig. 1A). WP_024273778 is most similar to colicin $\mathrm{Y}$ with $75 \%$ of amino acid identity between their pore forming domains (Fig. 1B,C). 


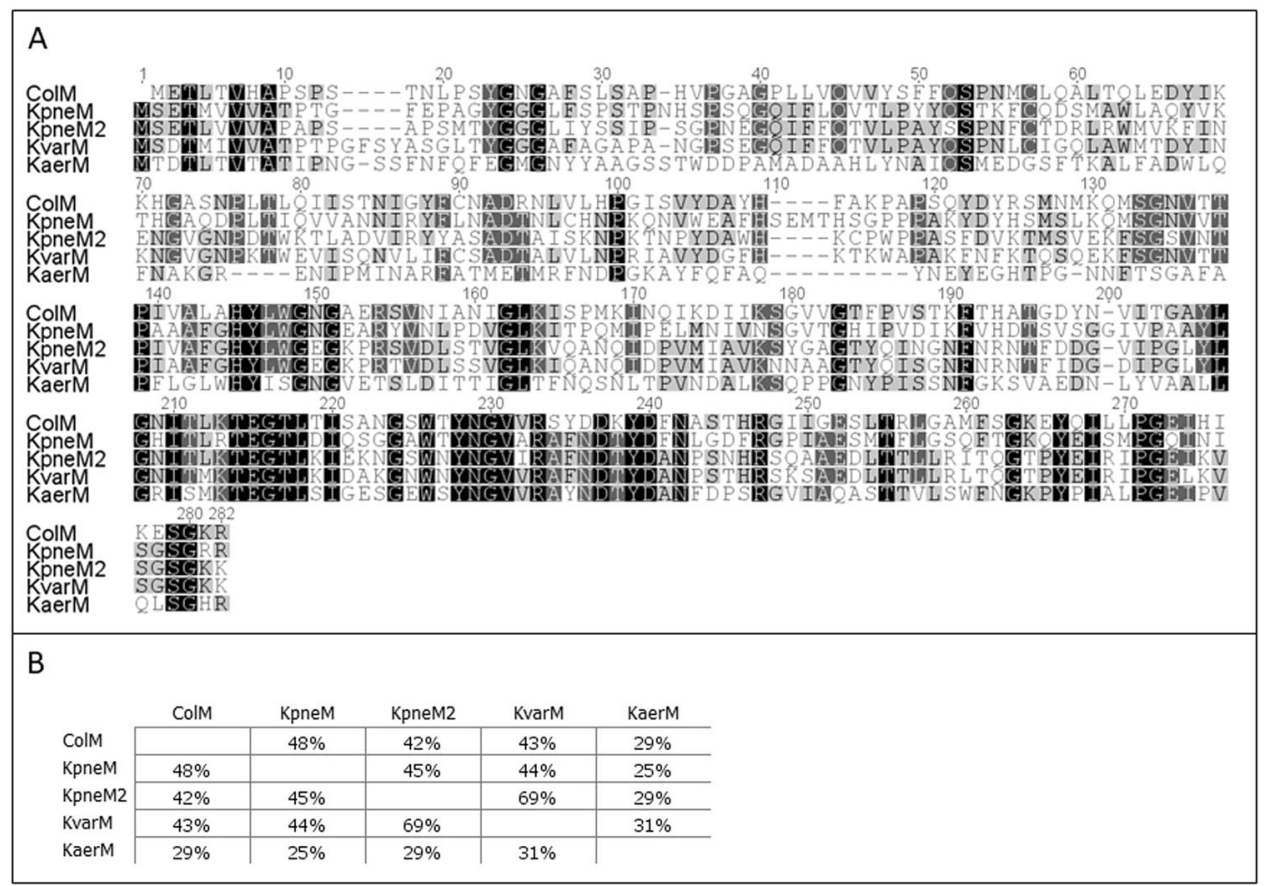

Figure 2. Sequence analysis of colicin $\mathrm{M}$ and its Klebsiella homologues. (A) Clustal W amino acid sequence alignments. ColM (AAA23589.1), KpneM (EWD35590.1), KpneM2 (WP_047066220), KvarM (CTQ17225.1), KaerM (WP_015367360.1).(B) Amino acid identity between colicin M and klebicins.

Based on these findings, we named putative Klebsiella bacteriocins according to the host species name and to the most related colicin: KpneA (K. pneumoniae SAV78255.1), KaerA (K. aerogenes WP_063414841.1), KoxyY (K. oxytoca WP_024273778), KvarIa (K. variicola KDL88409) and KpneIa (K. pneumoniae BAS34675). Amino acid sequences of predicted pore-forming domain of KpneIa and KvarIa are 52\% identical to ColIa (Fig. 1C,D). KpneIa and KvarIa are almost identical, but KpneIa contains a stretch of 52 a.a. in the central part of the protein, which is absent in KvarIa. KaerA and KpneA pore-forming domains have $46 \%$ and $42 \%$ identity with ColA, respectively (Fig. 1B,C).

The four colicin-M-like Klebsiella proteins show different degrees of homology with colicin M over full length of the protein in range of 29 to 48 percent of identity, the highest sequence similarity noticed in the carboxy-terminal domains (Fig. 2). All four proteins were also named according to the host species name: KpneM (K. pneumoniae EWD35590.1), KpneM2 (Klebsiella sp. WP_047066220), KvarM (K. variicola CTQ17225.1) and KaerM (K. aerogenes WP_015367360.1).

Klebicins are expressed in and recovered from green plants with high yield. All nine synthetic klebicin genes with plant-optimized codons, containing no added tags, were cloned into the magnICON ${ }^{\circledR}$ tobacco mosaic virus-based vector pICH29912 (Fig. 3A) ${ }^{19}$. The resultant binary expression vectors were used to transform A. tumefaciens. Klebicins were transiently expressed in plants by infiltrating transformed agrobacteria strains into leaves of young N. benthamiana plants by syringe infiltration. The SDS-PAGE and Coomassie staining analysis of the extracts of soluble proteins of infiltrated plant leaves revealed that all nine klebicins are efficiently expressed in plants and are detected in the gel as very intense supplementary bands (Fig. 3B). The weights of polypeptides observed in electrophoresis approximately correspond to the expected theoretical molecular weights (KvarIa 43.4 kDa, KpneIa - 48.5 kDa, KpneA - 40 kDa, Kaer A - 39 kDa, KoxyY - 48.7 kDa, KpneM - 30.3 kDa, KpneM2 $-29.7 \mathrm{kDa}$, KvarM - 29.8 kDa and KaerM - 29kDa) (Fig. 3B). The expression levels of individual klebicins varied in a range of 2,7-4,4 mg/g FW, the highest expression levels were achieved for the two K. pneumoniae M-type klebicins KpneM2 and KpneM (Table 1).

Plant-expressed klebicins exhibit broad antimicrobial activity against different Klebsiella species. As the next step, we tested the activity of the crude bacteriocin-containing plant extracts in soft-agar overlay assay with twelve Klebsiella strains belonging to different species (K. pneumoniae, K. quasipneumoniae, K. oxytoca, K. variicola and K. aerogenes). Two of the tested bacteriocins, KoxyY and KaerM demonstrated perceptible but very narrow inhibition zones on the lawn of several tested strains, with KaerM forming larger hazy inhibition zones only on K. aerogenes lawn. Because of the weak and/or narrow activity, these two proteins were not included in subsequent experiments (Fig. 4).

All seven remaining bacteriocins formed large inhibition zones on the lawn of several tested Klebsiella species and strains. All twelve tested strains were inhibited by several bacteriocins. The three ColM-like proteins demonstrated broadest activity spectrum and similar activity pattern, targeting eleven out of twelve tested strains. However, KvarM formed significantly larger inhibition zones than both K. pneumoniae ColM-like bacteriocins 


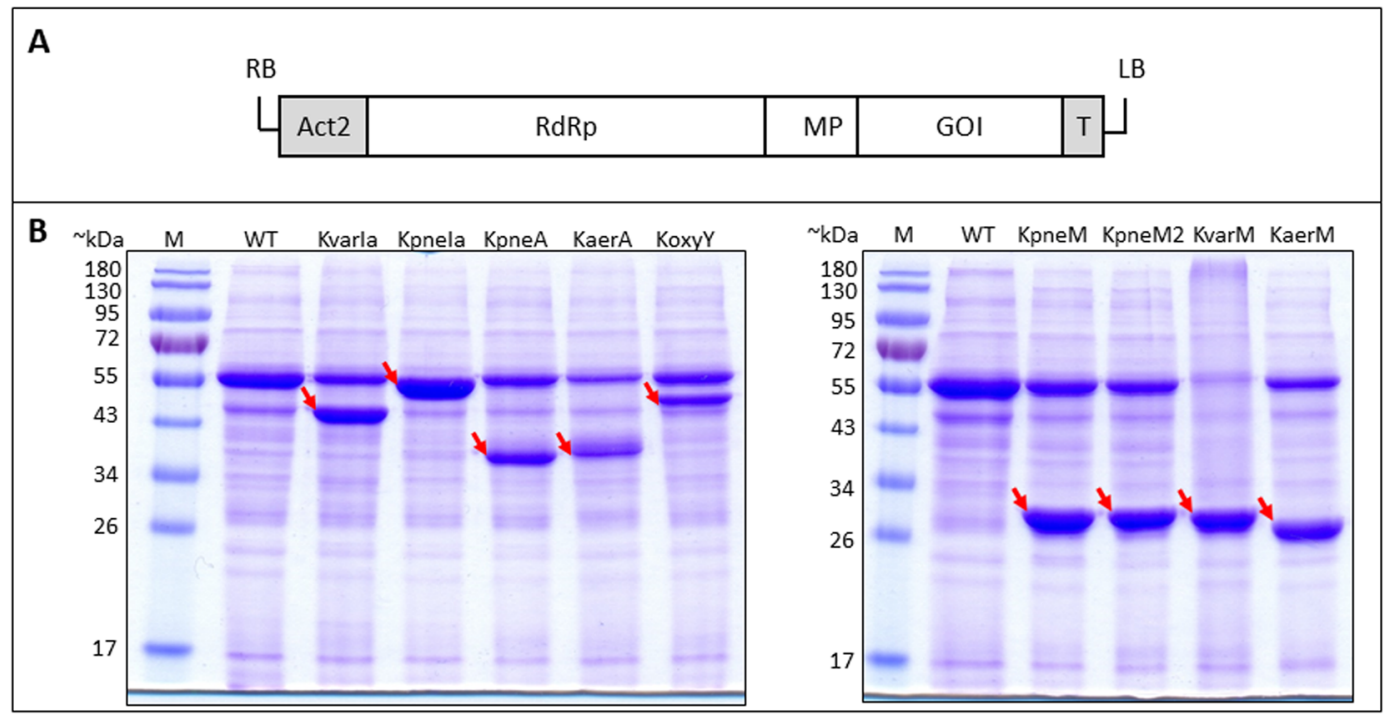

Figure 3. Klebicin expression in plants. (A) Schematic presentation of T-DNA region with klebicin expression cassette. $\mathrm{RB}$ - right T-DNA border, Act 2 - A. thaliana actin promoter, RdRp - RNA-dependent RNA polymerase, MP - tobacco mosaic virus movement protein, GOI - gene of interest, T - A. tumefaciens nopaline synthase terminator, LB - left T-DNA border. (B) Expression of klebicins in N. benthamiana leaves. Plant material ( $50 \mathrm{mg}$, pooled samples of three leaves) was harvested at 4 (KaerA) or 5 (all other klebicins) days post infiltration (dpi), ground in liquid nitrogen, extracted with $50 \mathrm{mM}$ Tris- $\mathrm{HCl}, 300 \mathrm{mM} \mathrm{NaCl} 15 \mathrm{mM}$ $\mathrm{CH}_{3} \mathrm{COONa}, 3 \mathrm{mM}$ DTT ( $\mathrm{pH} 7.5$ ), and denatured at $98^{\circ} \mathrm{C}$ for $10 \mathrm{~min}$. Solutions containing $5 \mu \mathrm{g}$ of protein were resolved in $12 \%$ polyacrylamide gel for Coomassie staining. $\mathrm{M}$ - PageRuler Prestained protein ladder (Thermo Fisher Scientific), WT - crude extract of non-infiltrated N. benthamiana leaves, KvarIa, KpneIa, KpneA, KaerA, KoxyY, KpneM, KpnM2, KvarA, KaerM - extracts of $N$. benthamiana leaves, infiltrated with klebicin expression constructs. Bands corresponding to recombinant klebicins are marked by arrows.

\begin{tabular}{|l|l|l|l|l|}
\hline Klebicin & $\begin{array}{l}\text { Purification } \\
\text { method }\end{array}$ & $\begin{array}{l}\text { Klebicin amount in } \\
\text { crude extract }(\mu \mathrm{g} / \mathrm{g} \text { FW })\end{array}$ & $\begin{array}{l}\text { Yield of purified } \\
\text { protein }(\mu \mathrm{g} / \mathrm{g} \text { FW })\end{array}$ & Purity \% \\
\hline KpneM & Phenyl > DS > Q & $3239 \pm 224$ & $1134 \pm 54$ & $99.8 \pm 0.3$ \\
\hline KpneM2 & Phenyl > DS > Q & $4448 \pm 347$ & $920 \pm 55$ & $99.5 \pm 0.5$ \\
\hline KvarM & Phenyl > DS > Q & $2423 \pm 146$ & $535 \pm 35$ & $98.1 \pm 1.0$ \\
\hline KpneA & Phenyl > DS > SP & $2677 \pm 163$ & $337 \pm 26$ & $97.2 \pm 1.2$ \\
\hline KaerA & SP > DS > Q & $2718 \pm 227$ & $468 \pm 46$ & $96.3 \pm 0.7$ \\
\hline KvarIa & Phenyl > DS > SP & $2697 \pm 149$ & $629 \pm 31$ & $98.9 \pm 1.0$ \\
\hline
\end{tabular}

Table 1. Purification method, obtained yields and purity of plant-expressed klebicins.

(KpneM and KpneM2). The two ColA-like proteins KpneA and KaerA also demonstrated a very similar activity pattern, although the zone diameter was different for some of the tested strains. And finally, both ColIa-like proteins KvarIa and KpneIa demonstrated an identical activity pattern (Fig. 4). All bacteriocins formed inhibition zones on the strains belonging to all five different Klebsiella species with exception of KvarIa and KpneIa. The two ColIa-like proteins had no effect on neither of four tested K. pneumoniae strains.

Thus, we confirmed that all cloned colicin-like proteins are indeed active Klebsiella colicin orthologues klebicins. This experiment with crude plant extracts allowed us to only semi-quantitatively compare activities of different klebicins because of differences in expression levels. However, it permitted us to choose the apparently most efficient bacteriocins for purification and further experiments: KpneA, KaerA, KvarIa, KpneM, KpneM2 and KvarM. KpneIa was not included in further experiments since it displays an identical spectrum as KvarIa.

Purification of klebicins. KpneA, KaerA, KvarIa, KpneM, KpneM2 and KvarM bacteriocins were purified to homogeneity by protein chromatography (up to $99.8 \%$ protein purity, Table 1). Quite pure KpneM, KpneM2 and KvarM proteins were obtained after single-step hydrophobic interaction chromatography (HIC), but for best results a second purification step by anion exchange chromatography was included. KpneA and KvarIa were also purified by HIC as a first step, but then followed by cation exchange chromatography. KaerA was purified by two steps of ion exchange chromatography, cation exchange column as a first step and anion exchange column as second step (Suppl. Text S1, Suppl. Figs S1, S2 and Table 1).

All purified klebicins contain only $0.2-3.7 \%$ of protein impurities, as determined by capillary gel electrophoresis. The yields of individual klebicins after purification are in range of $0.34-1.1 \mathrm{mg} / \mathrm{g}$ of fresh weight. The 


\begin{tabular}{|c|c|c|c|c|c|c|c|c|c|c|}
\hline Bacteriocin & WT & Kpne & Kpne & Kvar & Kaer & Kpne & Kaer & Koxy & Kvar & Kpne \\
\hline Strain & & & & & & & & & & \\
\hline $\begin{array}{l}\text { K. quasipneumoniae } \\
\text { DSM } 26371\end{array}$ & $\bullet$ & - & 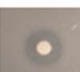 & 0 & 0 & 0 & $\bullet$ & - & - & $\bullet$ \\
\hline $\begin{array}{l}\text { K. pneumoniae } \\
\text { DSM } 789\end{array}$ & - & - & - & - & e & 0 & 0 & 0 & $\theta$ & - \\
\hline $\begin{array}{l}\text { K. pneumoniae } \\
\text { DSM } 9377\end{array}$ & • & $\bullet$ & - & 0 & 0 & e & $\bullet$ & - & e & - \\
\hline $\begin{array}{l}\text { K. pneumoniae } \\
\text { DSM } 16231\end{array}$ & • & $\bullet$ & e & - & - & 0 & e & $\rho$ & o & 0 \\
\hline $\begin{array}{l}\text { K. pneumoniae } \\
\text { DSM } 16358\end{array}$ & 0 & $\bullet$ & 0 & - & 0 & - & ○ & 0 & - & e \\
\hline $\begin{array}{l}\text { K. quasipneumoniae } \\
\text { DSM } 28211\end{array}$ & e & $\bullet$ & - & - & 0 & - & - & - & e & 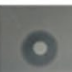 \\
\hline $\begin{array}{l}\text { K. quasipneumoniae } \\
\text { DSM } 28212\end{array}$ & 0 & - & - & - & 0 & 0 & - & 0 & - & - \\
\hline $\begin{array}{l}\text { K. oxytoca } \\
\text { DSM } 5175\end{array}$ & e & $\bullet$ & 0 & - & e & 0 & - & 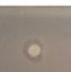 & - & e \\
\hline $\begin{array}{l}\text { K. oxytoca } \\
\text { DSM } 6673\end{array}$ & $\bullet$ & $\bullet$ & - & ○ & 0 & e & $\bullet$ & ○ & 0 & - \\
\hline $\begin{array}{l}\text { K. variicola } \\
\text { DSM } 15968\end{array}$ & 0 & $\bullet$ & $\bullet$ & - & - & - & $\bullet$ & e & e & - \\
\hline $\begin{array}{l}\text { K. aerogenes } \\
\text { DSM } 30053\end{array}$ & $\bullet$ & 0 & - & $\bullet$ & - & e & 0 & 0 & $\bullet$ & \\
\hline $\begin{array}{l}\text { K. aerogenes } \\
\text { DSM } 12058\end{array}$ & ○ & ○ & - & ? & $\bullet$ & 0 & 0 & o & - & \\
\hline
\end{tabular}

Figure 4. Evaluation of klebicin activity against Klebsiella strains in soft-agar overlay assay. Aliquots of $20 \mu \mathrm{l}$ $(\sim 50 \mu \mathrm{g})$ of protein crude extracts were spotted on $6 \mathrm{~mm}$ Whatman paper discs on Klebsiella lawn and incubated overnight.

purification of klebicins with greatest expression levels resulted in the biggest final yields and also best quality of purified proteins (highest purity) (Table 1).

We checked for the loss of the activity of purified klebicins by determining the minimal inhibitory concentrations (MIC) for two K. pneumoniae and K. variicola strains, tested with crude extracts in agar diffusion assays (Fig. 4). It appears that purified klebicins are highly active: for susceptible strains, MICs of the klebicins were in range of $0.1-0.8 \mu \mathrm{g} / \mathrm{ml}$ (Suppl. Table S1).

Klebicins as antimicrobial non-antibiotic alternative for control of MDR Klebsiella. As a next step, all six purified klebicins were tested against a larger panel of clinical Klebsiella isolates: 89 K. pneumoniae and $11 \mathrm{~K}$. oxytoca strains, in total one hundred clinical Klebsiella isolates from the Lithuanian Health Science University hospital in Kaunas. All isolates in the panel were resistant to at least one antibiotic and 68\% of isolates were multidrug resistant (resistant to three or more antimicrobial classes), but all were sensitive to carbapenems (Suppl. Table S2).

$92 \%$ in total and $88 \%$ of MDR isolates were sensitive to at least one klebicin. KvarM demonstrated the broadest spectrum of activity, since $85 \%$ of strains were sensitive to this klebicin (Fig. 5A and Suppl. Table S2). KpneM was not far behind, targeting $74 \%$ of tested strains, in general with slightly smaller inhibition zones. The specificity of KvarM and KpneM activity spectra were largely overlapping, but KvarM targeted 11 strains more that KpneM, and only one strain resistant to KvarM was sensitive to KpneM (Fig. 5A and Suppl. Table S2). In contrast, the third M-type klebicin KpneM2 was much less active, targeting only $20 \%$ of strains. Both ColA-like klebicins KpneA and KaerA targeted $30 \%$ and $28 \%$ of strains, respectively, with partially overlapping profiles. 9 strains resistant to KaerA were sensitive to KpneA, and 7 strains resistant to KpneA were sensitive to KaerA. KpneA also in general formed larger inhibition zones. KvarIa had the narrowest spectrum of activity and targeted only $10 \%$ of all strains, 6 strains of $K$. oxytoca and 4 strains of K. pneumoniae (Fig. 5A and Suppl. Table S2).

As the tested panel did not include carbapenem resistant strains, a separate study was done with NCTC antimicrobial resistance reference $K$. pneumoniae strains with different carbapenem resistance mechanisms (Fig. 5B). Despite the small strain panel in this test, the obtained data mirrors the results obtained with the panel of carbapenem-sensitive strains. KvarM and KpneM are active against all seven tested strains, KpneM2 forms 
A

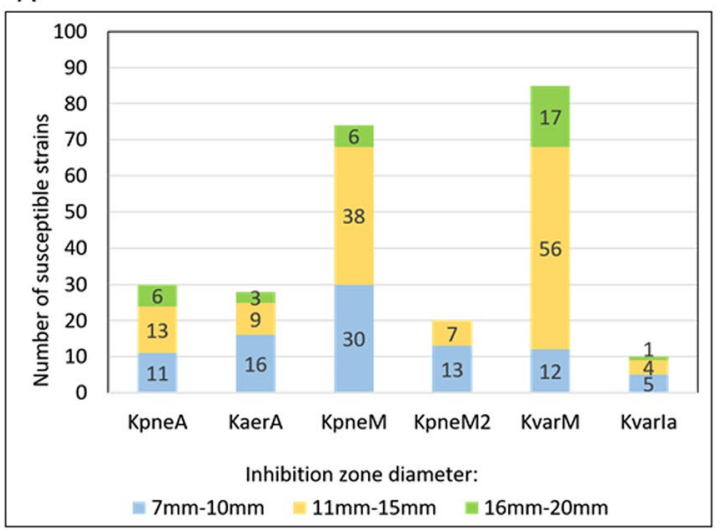

B

\begin{tabular}{|c|c|c|c|c|c|c|}
\hline $\begin{array}{l}\text { Carbapenem } \\
\text { resistance }\end{array}$ & $\begin{array}{l}\text { Bacteriocin/ } \\
\text { Strain }\end{array}$ & $\begin{array}{c}\text { Kpne } \\
\text { M }\end{array}$ & $\begin{array}{c}\text { Kpne } \\
\text { M2 }\end{array}$ & $\begin{array}{c}\text { Kvar } \\
\text { M }\end{array}$ & $\begin{array}{c}\text { Kpne } \\
\text { A }\end{array}$ & $\begin{array}{c}\text { Kaer } \\
\text { A }\end{array}$ \\
\hline \multirow[t]{3}{*}{$\begin{array}{l}\text { Class B } \\
\text { Carbapenemases }\end{array}$} & $\begin{array}{l}\text { K. pneumoniae } \\
\text { NCTC } 13439\end{array}$ & 0 & 0 & 0 & 0 & 0 \\
\hline & $\begin{array}{l}\text { K. pneumoniae } \\
\text { NCTC } 13440\end{array}$ & 0 & e & 0 & 0 & 0 \\
\hline & $\begin{array}{l}\text { K. pneumoniae } \\
\text { NCTC } 13443\end{array}$ & 0: & 웅 & 0 & e & - \\
\hline $\begin{array}{l}\text { Class D } \\
\text { Carbapenemases }\end{array}$ & $\begin{array}{l}\text { K. pneumoniae } \\
\text { NCTC } 13442\end{array}$ & 0 & 0 & 0 & 0 & 0 \\
\hline $\begin{array}{l}\text { Ambler class A } \beta \text { - } \\
\text { lactamase }\end{array}$ & $\begin{array}{l}\text { K. pneumoniae } \\
\text { NCTC } 13809\end{array}$ & 0 & 0 & 0 & o. & - \\
\hline Other mechanisms & $\begin{array}{l}\text { K. pneumoniae } \\
\text { NCTC } 13810\end{array}$ & 0 & Q & 0 & 0 & 9 \\
\hline $\begin{array}{l}\text { Class A } \\
\text { Carbapenemases }\end{array}$ & $\begin{array}{l}\text { K. pneumoniae } \\
\text { NCTC } 13438\end{array}$ & 0 & 0 & 0 & $\bullet$ & 0 \\
\hline
\end{tabular}

Figure 5. Sensitivity of clinical isolates to plant-expressed klebicins. (A) 100 clinical Klebsiella isolates (89 K. pneumoniae and $11 \mathrm{~K}$. oxytoca strains) were tested in soft agar overlay assay applying $20 \mu \mathrm{g}$ of each klebicin. The strains susceptible to each klebicin are grouped by the size of the inhibition zone. (B) Seven NCTC antimicrobial resistance reference K. pneumoniae strains with different mechanisms of carbapenem resistance were tested in soft agar overlay assay applying $20 \mu \mathrm{g}$ of each klebicin.

distinct inhibition zone on one strain and small hazy zones on several strains. KpneA targets three strains, and KaerA forms distinct zones on one strain.

Klebicins exhibit high antimicrobial activity on planktonic and biofilm cells of Klebsiella. We next performed a more detailed analysis of klebicin activity in liquid medium and on young, one day-old biofilms with five representatives of different Klebsiella species: K. pneumoniae, K. quasipneumoniae, K. oxytoca, K. variicola and K. aerogenes.

One best performing klebicin of each group (ColM-like, ColA-like and Colla-like) at a concentration of $5 \mu \mathrm{g} /$ $\mathrm{ml}$ was used in these assays. In liquid medium assay, klebicin KvarM inhibited the growth of all five strains, reducing the CFU number in quite similar extent - by four orders of magnitude for K. pneumoniae DSM 16231 and about three orders of magnitude for all remaining Klebsiellae. KvarIa inhibited four strains and was the most efficient of all three klebicins, reducing CFU by four to nine orders of magnitude, depending on the strain (as mentioned above, K. pneumoniae DSM16231 is insensitive to this klebicin). KpneA reduced CFU of three strains by 4.6 to 5.7 logs (Fig. 6A).

For biofilm assays, we used the same Klebsiella strains as in liquid culture assays. At first, we tested the ability of these five strains to form biofilms. Four of the tested strains formed biofilms in tested conditions, with exception of K. pneumoniae DSM 16231, thus this strain was not used for further experiments. The biofilms of all remaining four stains were treated for $20 \mathrm{~h}$ each with two klebicins, which showed the best results in liquid culture assays. The results obtained with biofilms quite closely reflected the results obtained in liquid culture assays, with exception of K. quasipneumoniae, for which biofilms were completely eradicated by KpneA and KvarIa. For all remaining strains, klebicin treatment efficiently decreased the numbers of CFU in biofilms to a similar extent as in liquid cultures (Fig. 6B).

Klebicins can rescue Galleria melonella from lethal Klebsiella infection. For the initial demonstration of klebicin activity in vivo, we have chosen to perform Klebsiella challenge assay in a non-mammal animal model, Galleria mellonella larvae. We selected KvarIa for this assay as one of most active klebicins and K. quasipneumoniae DSM 28212 as a KvarIa-sensitive challenge strain. At first, we determined the minimal lethal dose (MLD) of the bacterial challenge strain, sufficient to kill all the larvae within $68 \mathrm{~h}$ (the duration of experiment), as $2.3 \times 10^{4} \mathrm{CFU}$.

We next performed the challenge experiment with MLD and with two additional challenge doses, one inferior and one superior to MLD. $1.2 \times 10^{4} \mathrm{CFU}$ were not sufficient to kill all the larvae, as $15 \%$ of larvae still survived after $68 \mathrm{~h}$. However, 2.3 and $3.2 \times 10^{4} \mathrm{CFU}$ were sufficient to kill all the larvae within $44 \mathrm{~h}$. Injection of $10 \mu \mathrm{g}$ of KvarIa 2 hours post infection completely rescued all the larvae infected by 1.2 and $2.3 \times 10^{4}$ CFU. Larvae infected by the highest number of bacterial cells $\left(3.2 \times 10^{4} \mathrm{CFU}\right)$ were rescued partially, with $85 \%$ of larvae surviving until the end of experiment (Suppl. Fig. S3).

Identification of proteins exploited by klebicins for import into Klebsiella cells. In sharp contrast to some other bacteriocins, which are strictly species-specific (for example, pyocins), klebicin activity is not confined to the single species from which they are isolated, but rather to the genus. Having made this observation, we next inquired at the mechanisms involved in klebicin reception and translocation. Klebsiella quasipneumoniae DSM 28212, a strain with known genome sequence and sensitive to all the klebicins, was subjected to several rounds of transposon mutagenesis and pooled mutants were tested for their sensitivity towards different klebicins. 29 independent mutant clones were isolated, and transposon insertions were successfully mapped in 18 klebicin-resistant mutants. To confirm that klebicin sensitivity loss was indeed due to the mapped mutations, we performed complementation assays by ectopic expression of respective wild-type genes. The summarized 


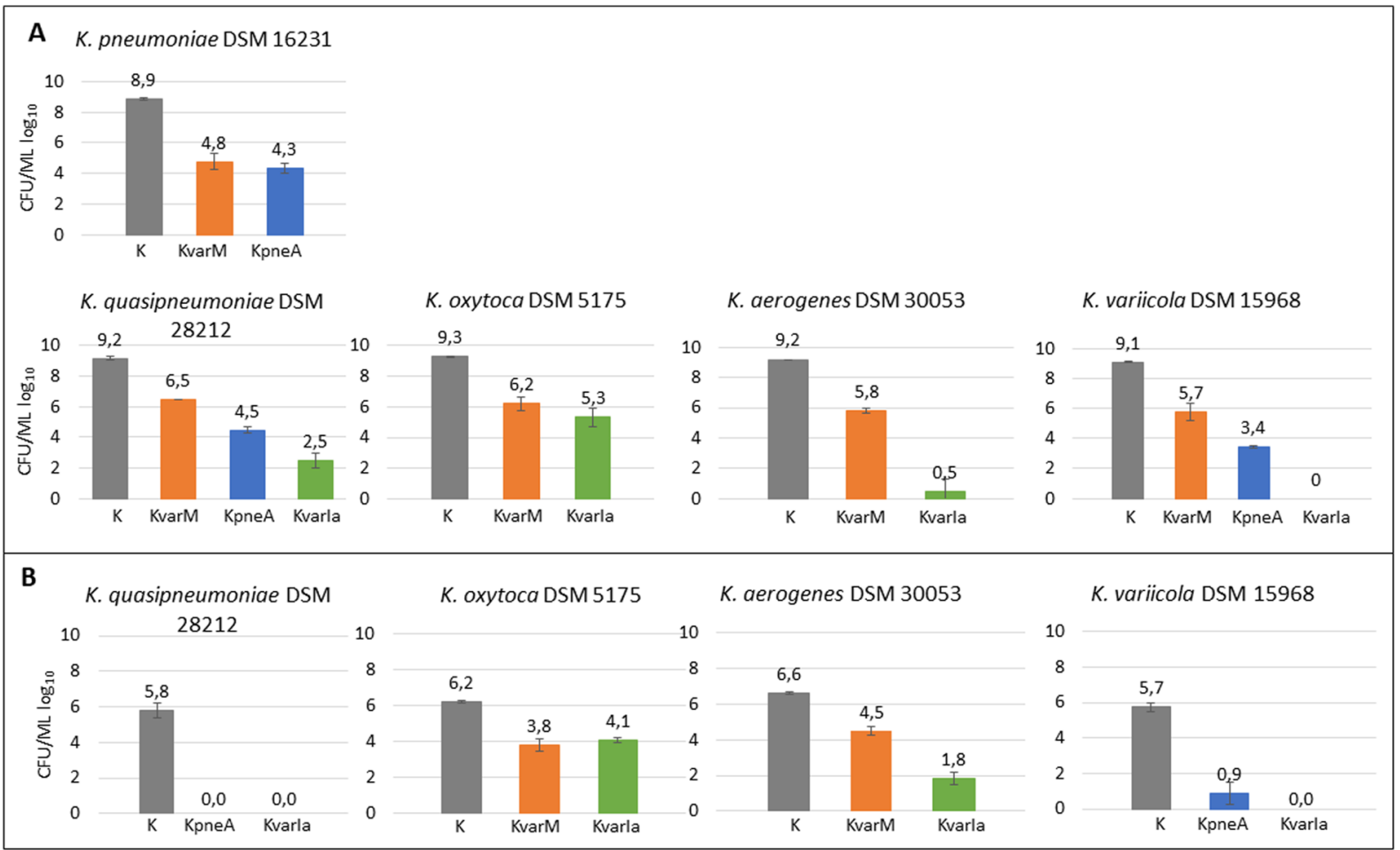

Figure 6. Evaluation of klebicin activity against Klebsiella in liquid cultures (A) and on biofilms (B). (A) Klebsiella overnight cultures were diluted to $\mathrm{OD}_{600}=0.3$ in CAA medium, then klebicins were added to bacterial cultures at $5 \mu \mathrm{g} / \mathrm{ml}$ and bacteria were further incubated for $5 \mathrm{~h}$ at $30^{\circ} \mathrm{C}$ or $37^{\circ} \mathrm{C}$ with shaking (200 rpm). (B) One day-old K. quasipneumoniae, K. oxytoca, K. variicola, K. aerogenes biofilms grown in CAA medium were treated with $5 \mu \mathrm{g} / \mathrm{ml}$ of either of klebicins (KvarM, KpneA, KvarIa represented by orange, blue and green bars, respectively) or buffer as control (K, shown in grey bars). At the end of experiments, serial dilutions were made, plated on LB agar plates, incubated at $28^{\circ} \mathrm{C}$ or $37^{\circ} \mathrm{C}$ for $24 \mathrm{~h}$ and $\mathrm{CFU}$ were enumerated. Data are the mean $\pm \mathrm{SD}$ of three independent experiments.

\begin{tabular}{|l|l|l|l|}
\hline Klebicin & Receptor & Mechanism of translocation & Presumptive cytotoxicity \\
\hline KpneM & FhuA & TonB, ExbB & Peptidoglycan synthesis inhibitor \\
\hline KpneM2 & FhuA & TonB, ExbB & Peptidoglycan synthesis inhibitor \\
\hline KvarM & FhuA & TonB, ExbB & Peptidoglycan synthesis inhibitor \\
\hline KpneA & OmpC & TonB, ExbB & Pore forming \\
\hline KaerA & OmpC & TonB, ExbB & Pore forming \\
\hline KvarIa & OmpC & $?$ & Pore forming \\
\hline
\end{tabular}

Table 2. Identified Klebsiella proteins involved in reception and translocation of klebicins.

results from sensitivity studies of klebicin mutants and complementation assays are presented in Supplementary Table S3.

Based on the obtained results, all klebicins with the exception of KvarIa are similar to group B colicins and use the TonB-dependent translocation pathway. All three M-type klebicins require ferrichrome receptor FhuA, TonB and ExbB for their reception - translocation, like their E. coli homologue colicin $\mathrm{M}^{7,20}$. Ferrichrome receptor in Pseudomonas FiuA is also the receptor of some Pseudomonas M-type pyocins ${ }^{21}$. KpneA and KaerA are also dependent on the TonB translocation pathway and they need in addition functional OmpC. So far, we could not identify any other putative receptor for these two klebicins (Table 2).

KvarIa-resistant transposon mutants were very difficult to obtain, and only some false-positive clones were isolated. Thus, we could identify only one protein participating in KvarIa reception - translocation, the outer membrane protein $\mathrm{C}(\mathrm{OmpC})$. OmpC mutants were selected by their resistance to KpneA and KaerA and it appeared that they are equally resistant to KvarIa.

\section{Discussion}

We successfully cloned nine colicin-like bacteriocins from several Klebsiella species. Based on sequence alignments, four of the cloned klebicins are homologs of colicin M, showing moderate to higher levels of sequence identity (29-48\%) with this protein. There was no published information about K. pneumoniae ColM-type bacteriocins when we started the study, but recently, Ghequire and co-workers ${ }^{18}$ have identified in Klebsiella genomes 
several ColM-like klebicins. Three ColM-like klebicins from our study are identical to klebicins from this independent study (KpneM is identical to Kpne CHS110, KpneM2 to Kpne e1602 and KvarM to Kvar 6A2).

Activity domains of the five remaining klebicins containing pore forming domains (pfam01024) demonstrate highest amino acid identities with colicin A (KpneA, KaerA), colicin Ia (KvarIa, KpneIa) and colicin Y (KoxyY).

Klebicin expression in plants is extremely efficient. Recently, expression of colicins and salmocins in plants have been reported ${ }^{13,22}$. The best expressed colicin (colK) amounted to $3 \mathrm{mg} / \mathrm{g} \mathrm{FW}$ and the best expressed salmocin (SalE2) reached $1.7 \mathrm{mg} / \mathrm{g}$ FW. For klebicins, the highest expression levels were as high as $4.4 \mathrm{mg} / \mathrm{g} \mathrm{FW}$ (KpneM2) and $3.3 \mathrm{mg} / \mathrm{g} \mathrm{FW}$ (KpneM).

The klebicin activity assays showed highly positive antibacterial effects. It appears that KvarM has an exceptionally wide spectrum of activity as it could target Klebsiella strains belonging to K. pneumoniae, K. quasipneumoniae, K. variicola, K. oxytoca, and K. aerogenes species. It was also active against $85 \%$ of strains in the panel of antibiotic-resistant clinical Klebsiella isolates. In liquid culture assay, this klebicin could reduce the colony forming number count by as much as three to four logs and more than by two logs in biofilm assay. Pore-forming klebicins, although more narrowly active, were in general even more efficient in reducing viability of planktonic bacteria or bacteria grown in biofilms than peptidoglycan synthesis inhibitors and were able to achieve four to nine logs of CFU reduction in liquid cultures and two to almost six logs of CFU reduction in biofilms. KvarIa, which has demonstrated highest efficiency in vitro, was also tested in vivo in G. mellonella larvae challenge assays with very good outcome. G. mellonella larvae model has been previously used to study the activity of pyocins S2 and $\mathrm{S} 5^{12,23}$. The G. mellonella model provides an excellent tool for pre-screening antimicrobial candidates and can help reduce the number of experiments with mammalian species ${ }^{12}$. Although it is not possible to compare directly the activity of KvarIa with activity of S2 or S5 in these different studies (different bacteria, different challenge doses and different bacteriocin doses were used), in all cases colicin-like bacteriocins efficiently protected larvae from death when used in range of $\sim 0.07-0.9 \mu \mathrm{mol} / \mathrm{kg}$ of body weight. However, the applicability of KvarIa is currently handicapped by the fact that it is not broadly active against K. pneumoniae, although it works well on its close relative K. quasipneumoniae. We thought that genetic engineering of this klebicin with the aim to expanding its specificity to include K. pneumoniae would be worthwhile to undertake. With this task in mind, we have started to analyse the translocation mechanisms that klebicins employ to cross the Klebsiella outer membrane in order to exert their cytotoxic functions.

A universal feature of all colicins is their domain organization, and each colicin appears to have receptor binding, translocation and cytotoxic domains, a feature that is conditioned by the necessity of these bacteriocins to cross the outer membrane of Gram-negative bacteria ${ }^{24}$. Amino acid sequence alignments of pore-forming klebicins with their E. coli counterparts reveals that their cytotoxic domains show a significant degree of homology. However, as a rule, pore forming klebicins have lower molecular weights than colicins. Their amino-terminal portions, which should contain translocation and receptor binding domains, are much shorter than the respective domains of colicins. Full-length protein amino sequence alignment between pore-forming colicins and klebicins demonstrates little sequence similarity outside cytotoxic domains. The exception is amino-terminal sequence of KvarIa, KpneIa and KoxyY (up to 45 first a.a.) highly identical to amino-terminal sequence of some group A pore-forming colicins (Suppl. Fig. 4). Thus, we anticipated that the translocation mechanism of pore-forming klebicins might be different than their E. coli counterparts.

In order to find klebicin-resistant mutants, we used mini transposon mutagenesis as a simple and rapid technique $^{25}$. We thus demonstrated that all three M-type klebicins KpneM, KpneM2 and KvarM are translocated by the mechanism similar to that of colicin $\mathrm{M}$ and that they need the FhuA receptor and TonB-related translocation pathway to enter the periplasm and exert their activity ${ }^{20,26}$.

Two klebicins, which we named KpneA and KaerA based on the similarity of their killing domain to colicin A, appeared also dependent on the TonB translocation pathway. This is in contrast to colicin A, which is translocated by TolA-dependent pathway ${ }^{27}$. Also, while colicin A binds to BtuB, we did not isolate any BtuB mutants resistant to KpneA or KaerA. However, both KpneA and KaerA need functional OmpC, an analog of OmpF, which participates also in ColA translocation ${ }^{24}$. We could not so far identify any other putative receptor for these two klebicins.

KvarIa is different from all remaining klebicins, as it appears to be functional in all TonB and ExbB mutants. Thus, based on our results, KvarIa does not use the TonB-dependent translocation pathway. Taking into account that all described colicins use either TonB or TolA as translocators, it would be expected that this protein is thus translocated by the Tol-dependent pathway. However, we did not isolate any single transpositional mutant of Tol-dependent pathway related genes that would be resistant to KvarIa. It certainly could be related to the limits of the method used, as KvarIa-resistant transposon mutants were very difficult to obtain and only some false-positive clones were isolated. Mutations with high fitness penalty might not have been obtained in the conditions used for selection. As a result, we could thus far identify only one protein participating in KvarIa reception - translocation, the outer membrane protein $\mathrm{C}(\mathrm{OmpC})$. OmpC mutants were selected by their resistance to KpneA and KaerA and it turned that they are equally resistant to KvarIa.

Further elucidation of klebicin receptors and translocators is important also for practical use of these klebicins. Klebicins are most promising to use for fighting antibiotic-resistant strains. Fighting carbapenem resistant strains would be most important and indeed all our tested carbapenem-resistant strains were sensitive to KvarM and KpneM. However, we had doubts that such strains will be targeted also by pore forming klebicins, as it has been shown that $97.1 \%$ of carbapenem-resistant Klebsiella strains do not express or express less OmpC or OmpF ${ }^{28}$. Thus, carbapenem-resistant Klebsiella could also be resistant to pore-forming klebicins, as all these klebicins require functional OmpC for their activity. However, three out of seven tested carbapenem-resistant strains were sensitive to KpneA, which uses OmpC as a receptor. These results exceeded our expectations, but testing a larger panel of carbapenem-resistant strains would be necessary to determine the real extent of their sensitivity to pore-forming klebicins. 
Further broad studies on resistance mutation frequencies are necessary to assess the practical utility of bacteriocins as antibiotic alternatives. Our data seem to indicate that the way forward would be the use of bacteriocin cocktails that combine proteins with different receptor/translocation routes, as well as the use of bacteriocins specifically engineered for low resistance frequency. Domains from different colicin-like bacteriocins can be combined to customize their features, such as target specificity, the immunity profile, mode of action and stability ${ }^{29}$. Taking advantage of publicly available genome sequence information, one could envisage designing bacteriocins with a high probability of hitting a chosen pathogen of interest by targeting receptors occurring frequently in nature, preferably essential for viability or resulting in a major growth disadvantages could spontaneous loss-of-function occur, and combined with toxin (sub)types for which immunity is rare ${ }^{30}$.

Currently, we can conclude that we have a panel of six highly efficient plant-expressed klebicins, which can together target over $94 \%$ (101 of 107) of Klebsiella clinical isolates evaluated. Even without further engineering and improvement, these proteins could be further developed as an antimicrobial cocktail for their potential use against antibiotic-resistant Klebsiella.

\section{Methods}

Bacterial strains and cultures. Klebsiella strains were purchased from Leibniz Institute DSMZ-German Collection of Microorganisms and Cell Cultures and from Public Health England National Collection of Type Cultures (NCTC) and are described in Supplementary Information (Suppl. Table S4). Unless otherwise stated, Klebsiella strains were prepared by culturing in Lysogeny Broth (LB) medium (Roth) or Casamino Acids (CAA) $\left(0.5 \%\right.$ Bacto $^{\mathrm{TM}}$ Casamino acids, $5.2 \mathrm{mM} \mathrm{K}_{2} \mathrm{HPO}_{4}, 1 \mathrm{mM} \mathrm{MgSO}_{4}$ ) medium (BD Biosciences) at $28^{\circ} \mathrm{C}, 30^{\circ} \mathrm{C}$ or $37^{\circ} \mathrm{C}$ (as indicated in Suppl. Table 1) with shaking (200 rpm); overnight cultures were prepared by inoculation from frozen stocks. Clinical Klebsiella strains used for agar overlay assay have been isolated in Lithuanian University of Health Sciences, Kaunas clinics, and are described in Suppl. Table S2.

Construction of klebicin expression vectors. The open reading frames encoding for KpneA (K. pneumoniae SAV78255.1), KaerA (K. aerogenes WP_063414841.1), KoxyY (K. oxytoca WP_024273778), KvarIa (K. variicola KDL88409), KpneIa (K. pneumoniae BAS34675), KpneM (K. pneumoniae EWD35590.1), KpneM2 (Klebsiella sp. WP_047066220), KvarM (K. variicola CTQ17225.1), and KaerM (K. aerogenes WP_015367360.1) optimized for expression in the host plant Nicotiana benthamiana were synthetized by Thermofisher Scientific (USA) and inserted as BsaI-BsaI fragments into pICH29912, an assembled TMV-based magnICON ${ }^{\circledR}$ vector $^{19}$ (Fig. 3A). Obtained plasmids were used to transform A. tumefaciens GV3101.

Klebicin expression in plants. Klebicin expression in plants was performed as described in ${ }^{12}$ with some modifications. (Nicotiana benthamiana plants were grown in a growth chamber at $25^{\circ} \mathrm{C}$ and $50 \%$ humidity, with a $16 \mathrm{~h}$ light (1500 lux) and $8 \mathrm{~h}$ dark photoperiod. Four-to-six-week-old plants were used for transfection with recombinant $A$. tumefaciens.

A. tumefaciens were grown overnight at $30^{\circ} \mathrm{C}$ in $\mathrm{LB}$ medium containing $50 \mathrm{mg} / \mathrm{l} \mathrm{rifampicin} \mathrm{and} 50 \mathrm{mg} / \mathrm{l} \mathrm{kana-}$ mycin. Agrobacterium overnight cultures were sedimented at $3220 \mathrm{~g}$ for $5 \mathrm{~min}$ and resuspended in infiltration buffer (10 mM MES, $10 \mathrm{mM} \mathrm{MgSO}_{4}$, pH 5.5) at an $\mathrm{OD}_{595}$ of 1.5.

Four-to-six-week-old plant leaves were infiltrated into the abaxial side of the leaf using a syringe without a needle with a 1:100 dilution of $A$. tumefaciens strain containing the expression vector for each klebicin. Plant leaves were observed and collected at 4-7 dpi (days post infiltration).

Purification of plant-produced klebicins. Preparation of crude extracts of plant-produced klebicins was performed as described in ${ }^{12}$ with some modifications. A small portion of frozen leaf tissue was ground into fine powder with mortar and pestle using liquid nitrogen. Prepared powder was mixed with cold extraction buffer at a ratio of $1 \mathrm{~g}$ of plant material to $5 \mathrm{ml}$ of buffer. The suspension was kept on ice for $15-20 \mathrm{~min}$. Cell debris was removed by centrifugation at $3220 \mathrm{~g}$ at $4^{\circ} \mathrm{C}$ for $20 \mathrm{~min}$., and the supernatant was filtered through membrane filters (pore sizes $5 \mu \mathrm{m}$ and $0.22 \mu \mathrm{m}$ ). Obtained solution was taken as total soluble protein and applied for purification by two-step chromatography. Detailed protocols of purification are presented in Supplementary information (Suppl. Text 1 and Suppl. Fig. 1). The concentration of purified proteins was evaluated by Bradford assay or by comparison of band intensity with known BSA amount run on the same SDS-PAGE gel.

Klebicin antimicrobial activity evaluation in liquid culture. Klebicin antimicrobial activity evaluation was performed as described by ${ }^{12}$, with some modifications. Klebsiella overnight cultures were diluted to $\mathrm{OD}_{595}=0.3$ in iron-deficient Casamino Acids (CAA) medium (BD Bioscience). Lyophilized purified klebicins were resuspended in CAA medium, added to diluted bacterial suspension and incubated for 5 hours at $30^{\circ} \mathrm{C}$ or $37^{\circ} \mathrm{C}$ with shaking $(200 \mathrm{rpm})$. The antimicrobial activity of klebicins was evaluated by determining cell numbers of bacterial test culture. Serial 10-fold dilutions, $10^{-1}, 10^{-2}, 10^{-3}, 10^{-4}$ and $10^{-5}$ were made, plated on LB agar plates. Upon incubation of plates for overnight at $30^{\circ} \mathrm{C}$ or $37^{\circ} \mathrm{C}, \mathrm{CFU}$ were counted and cell numbers per $\mathrm{ml}$ of bacterial culture were calculated. All experiments were repeated 3 times.

Soft-agar overlay assays. Soft-agar overlay assays were performed as described by ${ }^{12}$, with some modifications. Klebsiella overnight cultures were equalized to $\mathrm{OD}_{595}=1.0$ in $\mathrm{LB}$ medium and diluted 100 -fold in $0.8 \%$ $(\mathrm{w} / \mathrm{v})$ top agar preheated in a $55^{\circ} \mathrm{C}$ water bath. Mixed overlay components were poured on plates containing solid medium ( $\mathrm{LB}$ containing $1.5 \%(\mathrm{w} / \mathrm{v})$ agar). Sterile Whatman paper discs (6 $\mathrm{mm}$ diameter) were placed on the surface of the soft-agar medium containing bacterial test strains and respective amounts of klebicins $(20 \mu \mathrm{l}$ of crude extracts or $10 \mu \mathrm{g}$ of purified klebicins) were applied to the discs. The plates were incubated for overnight at $30^{\circ} \mathrm{C}$ or $37^{\circ} \mathrm{C}$ and the diameter of klebicin inhibition zones was measured. 
Biofilm assays. Biofilms were grown as described by ${ }^{12,31}$, with some modifications. Briefly, K. quasipneumoniae, $K$. oxytoca, K. variicola, K. aerogenes strains were grown overnight in LB and diluted to $\mathrm{OD}_{595}=0.1$ with fresh CAA medium. For preparation of the biofilm growth plate, $10 \mu \mathrm{l}$ of bacterial culture were transferred to the wells of a 96-well microtiter plate (Nalgene Nunc International, Rochester, N.Y.) containing $90 \mu \mathrm{l}$ of CAA medium. Bacterial biofilms were formed by immersing the pegs of the biofilm detection lid, a modified polystyrene microtiter lid (Nunc TSP system), into the wells of the biofilm growth plate, followed by incubation at $30^{\circ} \mathrm{C}$ or $37^{\circ} \mathrm{C}$ for $20 \mathrm{~h}$. For treatment with klebicins, peg biofilm detection lids were rinsed three times in sterile water, placed into microtiter plates containing $5 \mu \mathrm{g} / \mathrm{ml}$ of klebicins diluted in $100 \mu \mathrm{l} \mathrm{CAA} \mathrm{per} \mathrm{well} \mathrm{and} \mathrm{incubated} \mathrm{for} 5 \mathrm{~h}$ at $30^{\circ} \mathrm{C}$ or $37^{\circ} \mathrm{C}$, depending on the strain. After incubation with klebicins, peg lids were again rinsed three times in sterile water, placed into CAA in a sterile microtiter plate and centrifuged at $810 \mathrm{~g}$ for $30 \mathrm{~min}$. The content of 6 identically treated wells was pooled for each sample and serial dilutions thereof were made and plated on LB plates for CFU enumeration. All experiments were repeated 3 times.

Galleria mellonella larvae challenge experiments. Galleria mellonella challenge experiments were performed as described in ${ }^{12}$, with some modifications. Klebsiella quasipneumoniae strain DSM 28212 culture was grown for overnight in $\mathrm{LB}$ medium and diluted in $0.8 \%(\mathrm{w} / \mathrm{v}) \mathrm{NaCl}$ in order to achieve a concentration of 1.2$3.2 \times 10^{6} \mathrm{CFU} / \mathrm{ml}$. $10 \mu \mathrm{l}$ of $\mathrm{K}$. quasipneumoniae culture and $10 \mu \mathrm{l}$ of klebicin solution $(1 \mu \mathrm{g} / \mu \mathrm{l})$ were injected into the hemocoel of the fifth instar of G. mellonella larvae (Livefood UK) in proximity of the left and/or right prolegs. Klebicins were injected two hours post infection with $K$. quasipneumoniae. Injected larvae were incubated at $37^{\circ} \mathrm{C}$ in $9 \mathrm{~cm}$ Petri dishes without food for up to 3 days. Caterpillars were considered dead when they displayed no movement in response to mechanical stimulus to the head, leading to distinct change in color from cream to dark brown/black. Twenty larvae were used per each treatment point.

Transposon mutagenesis of $K$. quasipneumoniae DSM 28212. Transposon mutagenesis of K. quasipneumoniae strain DSM 28212 was performed as described in ${ }^{25}$. The suicide delivery of mini-transposons localized in pBAM1 plasmid was performed by triparental mating. The plasmid was mobilized from E. coli CC118 $\lambda$ pir (pBAM1) donor cells into K. quasipneumoniae DSM 28212 cells with the assistance of the helper strain E. coli HB101 (pRK600). Obtained kanamycin-resistant clones were confirmed for the loss of the ampicillin resistance and their genomic DNA was used for the PCR amplification of the transposon adjacent regions, followed by sequencing, as described by Martínez-García and co-workers ${ }^{25}$.

Complementation assays. Klebsiella genome regions, containing ExbB, ExbBD, OmpC, FhuA, TonB and FimB gene ORFs along with $5^{\prime}$ non-coding promoter regions, were PCR-amplified from K. quasipneumoniae DSM 28212 genomic DNA with help of Phusion DNA polymerase (Thermofisher Scientific Baltics) and ligated in pJET1.2 (Thermofisher Scientific Baltics). After sequencing, cloned fragments were excised with a pair of restriction endonucleases specific for each fragment, ligated in pACYC184 (NEB) and transformed into the respective K. quasipneumoniae mutants. The sequences of primers used and cloning strategy are described in Suppl. Table S5.

\section{Data availability}

All data generated or analysed during this study are included in this published article and Supplementary Information.

Received: 4 July 2019; Accepted: 9 October 2019;

Published online: 28 October 2019

\section{References}

1. Podschun, R. \& Ullmann, U. Klebsiella spp. as nosocomial pathogens: epidemiology, taxonomy, typing methods, and pathogenicity factors. Clin Microbiol Rev 11, 589-603 (1998).

2. Long, S. W. et al. Whole-Genome Sequencing of Human Clinical Klebsiella pneumoniae Isolates Reveals Misidentification and Misunderstandings of Klebsiella pneumoniae, Klebsiella variicola, and Klebsiella quasipneumoniae. mSphere 2, https://doi. org/10.1128/mSphereDirect.00290-17 (2017).

3. Miftode, E. et al. Antimicrobial resistance profile of E. coli and Klebsiella spp. from urine in the Infectious Diseases Hospital Iasi. Rev Med Chir Soc Med Nat Iasi 112, 478-482 (2008).

4. Weisenberg, S. A., Morgan, D. J., Espinal-Witter, R. \& Larone, D. H. Clinical outcomes of patients with Klebsiella pneumoniae carbapenemase-producing K. pneumoniae after treatment with imipenem or meropenem. Diagn Microbiol Infect Dis 64, 233-235, https://doi.org/10.1016/j.diagmicrobio.2009.02.004 (2009).

5. Rice, L. B. Federal funding for the study of antimicrobial resistance in nosocomial pathogens: no ESKAPE. J Infect Dis 197, 1079-1081, https://doi.org/10.1086/533452 (2008).

6. Gu, D. et al. A fatal outbreak of ST11 carbapenem-resistant hypervirulent Klebsiella pneumoniae in a Chinese hospital: a molecular epidemiological study. Lancet Infect Dis 18, 37-46, https://doi.org/10.1016/S1473-3099(17)30489-9 (2018).

7. Braun, V., Gunter, K. \& Hantke, K. Transport of iron across the outer membrane. Biol Met 4, 14-22 (1991).

8. Scholl, D. et al. An engineered R-type pyocin is a highly specific and sensitive bactericidal agent for the food-borne pathogen Escherichia coli O157:H7. Antimicrob Agents Chemother 53, 3074-3080, https://doi.org/10.1128/AAC.01660-08 (2009).

9. Brown, C. L., Smith, K., McCaughey, L. \& Walker, D. Colicin-like bacteriocins as novel therapeutic agents for the treatment of chronic biofilm-mediated infection. Biochem Soc Trans 40, 1549-1552, https://doi.org/10.1042/BST20120241 (2012).

10. Grinter, R., Milner, J. \& Walker, D. Beware of proteins bearing gifts: protein antibiotics that use iron as a Trojan horse. FEMS Microbiol Lett 338, 1-9, https://doi.org/10.1111/1574-6968.12011 (2013).

11. Ghequire, M. G. \& De Mot, R. Ribosomally encoded antibacterial proteins and peptides from Pseudomonas. FEMS Microbiol Rev 38, 523-568, https://doi.org/10.1111/1574-6976.12079 (2014).

12. Paskevicius, S. et al. Plant-expressed pyocins for control of Pseudomonas aeruginosa. PLoS One 12, e0185782, https://doi. org/10.1371/journal.pone.0185782 (2017). 
13. Schulz, S. et al. Broad and efficient control of major foodborne pathogenic strains of Escherichia coli by mixtures of plant-produced colicins. Proc Natl Acad Sci USA 112, E5454-5460, https://doi.org/10.1073/pnas.1513311112 (2015).

14. Cascales, E. et al. Colicin biology. Microbiol Mol Biol Rev 71, 158-229, https://doi.org/10.1128/MMBR.00036-06 (2007).

15. James, R., Schneider, J. \& Cooper, P. C. Characterization of three group A klebicin plasmids: localization of their E colicin immunity genes. J Gen Microbiol 133, 2253-2262, https://doi.org/10.1099/00221287-133-8-2253 (1987).

16. Riley, M. A., Pinou, T., Wertz, J. E., Tan, Y. \& Valletta, C. M. Molecular characterization of the klebicin B plasmid of Klebsiella pneumoniae. Plasmid 45, 209-221, https://doi.org/10.1006/plas.2001.1519 (2001).

17. Chavan, M., Rafi, H., Wertz, J., Goldstone, C. \& Riley, M. A. Phage associated bacteriocins reveal a novel mechanism for bacteriocin diversification in Klebsiella. J Mol Evol 60, 546-556, https://doi.org/10.1007/s00239-004-0263-9 (2005).

18. Ghequire, M. G. K., Buchanan, S. K. \& De Mot, R. The ColM Family, Polymorphic Toxins Breaching the Bacterial Cell Wall. MBio 9, https://doi.org/10.1128/mBio.02267-17 (2018).

19. Marillonnet, S., Thoeringer, C., Kandzia, R., Klimyuk, V. \& Gleba, Y. Systemic Agrobacterium tumefaciens-mediated transfection of viral replicons for efficient transient expression in plants. Nat Biotechnol 23, 718-723, https://doi.org/10.1038/nbt1094 (2005).

20. Braun, V., Helbig, S. \& Patzer, S. I. Import of periplasmic bacteriocins targeting the murein. Biochem Soc Trans 40, 1449-1455, https://doi.org/10.1042/BST20120175 (2012).

21. Ghequire, M. G., Kemland, L., Anoz-Carbonell, E., Buchanan, S. K. \& De Mot, R. A Natural Chimeric Pseudomonas Bacteriocin with Novel Pore-Forming Activity Parasitizes the Ferrichrome Transporter. MBio 8, https://doi.org/10.1128/mBio.01961-16 (2017).

22. Schneider, T. et al. Plant-made Salmonella bacteriocins salmocins for control of Salmonella pathovars. Sci Rep 8, 4078, https://doi. org/10.1038/s41598-018-22465-9 (2018).

23. Smith, K. et al. Activity of pyocin S2 against Pseudomonas aeruginosa biofilms. Antimicrob Agents Chemother 56, 1599-1601, https://doi.org/10.1128/AAC.05714-11 (2012).

24. Kleanthous, C. Swimming against the tide: progress and challenges in our understanding of colicin translocation. Nat Rev Microbiol 8, 843-848, https://doi.org/10.1038/nrmicro2454 (2010).

25. Martinez-Garcia, E., Calles, B., Arevalo-Rodriguez, M. \& de Lorenzo, V. pBAM1: an all-synthetic genetic tool for analysis and construction of complex bacterial phenotypes. BMC Microbiol 11, 38, https://doi.org/10.1186/1471-2180-11-38 (2011).

26. Helbig, S. \& Braun, V. Mapping functional domains of colicin M. J Bacteriol 193, 815-821, https://doi.org/10.1128/JB.01206-10 (2011).

27. Kim, Y. C., Tarr, A. W. \& Penfold, C. N. Colicin import into E. coli cells: a model system for insights into the import mechanisms of bacteriocins. Biochim Biophys Acta 1843, 1717-1731, https://doi.org/10.1016/j.bbamcr.2014.04.010 (2014).

28. Ye, Y. et al. Mechanism for carbapenem resistance of clinical Enterobacteriaceae isolates. Exp Ther Med 15, 1143-1149, https://doi. org/10.3892/etm.2017.5485 (2018).

29. Behrens, H. M., Walker, S. A. \& Kleanthous, D. The therapeutic potential of bacteriocins as protein antibiotics. Emerging Topics in Life Sciences 1, 65-74 (2017).

30. Ghequire, M. G. K. \& De Mot, R. Turning Over a New Leaf: Bacteriocins Going Green. Trends Microbiol 26, 1-2, https://doi. org/10.1016/j.tim.2017.11.001 (2018).

31. Moskowitz, S. M., Foster, J. M., Emerson, J. \& Burns, J. L. Clinically feasible biofilm susceptibility assay for isolates of Pseudomonas aeruginosa from patients with cystic fibrosis. J Clin Microbiol 42, 1915-1922, https://doi.org/10.1128/jcm.42.5.1915-1922.2004 (2004).

\section{Acknowledgements}

We thank Dr. Daniel Tusé for critical reading of the manuscript. The work was supported by the Lithuanian Business Support Agency grant (Grant Number J05-LVPA-K-03-0011).

\section{Author contributions}

E.D., Š.P., A.M., B.S., U.S., S. H.-L., S.S. and A.V. conceived and conducted the experiments, Y.G. and A.G. analysed the results, A.R. conceived the experiments and wrote the manuscript. All authors reviewed the manuscript.

\section{Competing interests}

Nomad Bioscience GmbH and Nomads UAB have filed a patent on the use of klebicins to control Klebsiella.

\section{Additional information}

Supplementary information is available for this paper at https://doi.org/10.1038/s41598-019-51969-1.

Correspondence and requests for materials should be addressed to A.R.

Reprints and permissions information is available at www.nature.com/reprints.

Publisher's note Springer Nature remains neutral with regard to jurisdictional claims in published maps and institutional affiliations.

Open Access This article is licensed under a Creative Commons Attribution 4.0 International License, which permits use, sharing, adaptation, distribution and reproduction in any medium or format, as long as you give appropriate credit to the original author(s) and the source, provide a link to the Creative Commons license, and indicate if changes were made. The images or other third party material in this article are included in the article's Creative Commons license, unless indicated otherwise in a credit line to the material. If material is not included in the article's Creative Commons license and your intended use is not permitted by statutory regulation or exceeds the permitted use, you will need to obtain permission directly from the copyright holder. To view a copy of this license, visit http://creativecommons.org/licenses/by/4.0/.

(C) The Author(s) 2019 